\title{
Two-Dimensional Melting of Colloidal Hard Spheres
}

\author{
Alice L. Thorneywork, Joshua L. Abbott, Dirk G. A. L. Aarts and Roel P. A. Dullens \\ Department of Chemistry, Physical and Theoretical Chemistry Laboratory, \\ University of Oxford, South Parks Road, Oxford OX1 3QZ, United Kingdom
}

\begin{abstract}
We study the melting of quasi-two-dimensional colloidal hard spheres by considering a tilted monolayer of particles in sedimentation-diffusion equilibrium. In particular, we measure the hard-disk equation of state from the density profiles and use time-dependent and height-resolved correlation functions to identify the liquid, hexatic and crystal phases. We find that the liquid-hexatic transition is first order and that the hexatic-crystal transition is continuous. Furthermore, we directly measure the width of the liquid-hexatic coexistence gap from the fluctuations of the corresponding interface, and thereby experimentally establish the full phase behaviour of hard disks.
\end{abstract}

Thermal hard spheres are the fruit flies of statistical mechanics as they embody the simplest interacting many-body system. In the 1950s, signs of a purely entropic first-order freezing transition in three-dimensional (3D) hard spheres were observed in simulations $[1,2]$, but led to much controversy [3], only resolved 30 years later when the numerical predictions were tested and confirmed by experiments using colloidal model hard spheres [4]. Surprisingly, it has taken much longer for simulations to converge on a prediction for the nature of the freezing transition of spherical particles in a twodimensional (2D) geometry, making the phase behaviour of the 2D analogue, hard disks, the subject of substantial debate [5-14]. Recent simulations have suggested, however, that hard disks undergo a first-order transition from the isotropic liquid to the so-called hexatic phase, and a continuous transition from the hexatic phase to the crystalline phase $[11,12]$.

For 2D systems in general, melting via the hexatic phase is rationalised by the celebrated KosterlitzThouless-Halperin-Nelson-Young (KTHNY) scenario [15-18], introduced in the 1970s. This relates the increasing disorder upon melting of $2 \mathrm{D}$ crystals to the unbinding of topological defects. More specifically, the unbinding of dislocation pairs into free dislocations first transforms the 2D crystal, characterised by long-ranged bond-orientational and quasi-long-ranged translational order, into the hexatic phase. Importantly, while the free dislocations destroy the translational order in the hexatic phase, the bond-orientational order is retained. The hexatic phase subsequently becomes a liquid by the unbinding of dislocations into isolated disclinations, resulting in the liquid exhibiting both short-ranged bond-orientational and translational order.

Many aspects of KTHNY theory have been observed in experiments on 2D systems of super-paramagnetic colloids interacting via a soft potential [19-23]. However, results from simulations suggest that the nature of $2 \mathrm{D}$ melting may in fact be sensitively affected by the pair potential [14, 24], particle shape [25], out-of plane fluctuations [13], pinned particles [26, 27] or vacancies [28], with alternative melting scenarios seen experimentally in different colloidal systems [29-32]. Nevertheless, for the simplest possible interacting system in $2 \mathrm{D}$, hard disks, the nature of the melting transition is yet to be confirmed experimentally - more than half a century after the first simulations of hard-disk freezing were published - despite receiving an enormous amount of attention [5-14].

Here, we use a tilted monolayer of colloidal hard spheres in sedimentation-diffusion equilibrium to experimentally establish the full phase behaviour of hard disks. Our quasi-2D colloidal system has previously been shown to be an excellent model for hard disks $[35,36]$. It is prepared by allowing $2.79 \mu \mathrm{m}$ diameter melamine formalydehyde spheres in a water/ethanol mixture to sediment, to form a monolayer at the base of a glass sample cell (see Supplemental Material [37]). The samples are placed on a bright-field video-microscope, which is tilted by a small and variable angle, $\alpha$. This leads to a further sedimentation of the particles parallel to the base of the cell, which after equilibration for many weeks establishes an in-plane sedimentation-diffusion equilibrium (see Fig. 1a). Importantly, the values of $\alpha$ are carefully chosen such that the gravitational height parallel $\left(h_{g \|}\right)$ to the base of the cell is large, resulting in an area fraction gradient as a function of the height $z, \phi(z)$. At the same time, the gravitational height perpendicular $\left(h_{g \perp}\right)$ to the base of the cell is less than $3 \%$ of the particle diameter at all tilt angles, ensuring the 2D nature of our system at all area fractions (see Supplemental Material [37]). Following equilibration, the system is imaged at a rate of one frame per second for two hours, and standard particle tracking procedures [38] are used to obtain particle coordinates and tracks. Note that in the following discussion, the coordinate axes are defined such that the $z$-axis runs parallel to the $\phi(z)$ gradient and the $x$-axis perpendicular to it.

Figure 1a shows a typical experimental image of the colloidal monolayer in sedimentation-diffusion equilibrium at a tilt angle $\alpha=0.56^{\circ}$. Here, the structure is seen to vary from that of a dilute liquid at large $z$ to a dense ordered phase at small $z$. Crucially, the width of the region over which this transition occurs increases with decreasing tilt angle, allowing for a detailed investigation of the full phase behaviour of the system (see 
(a)
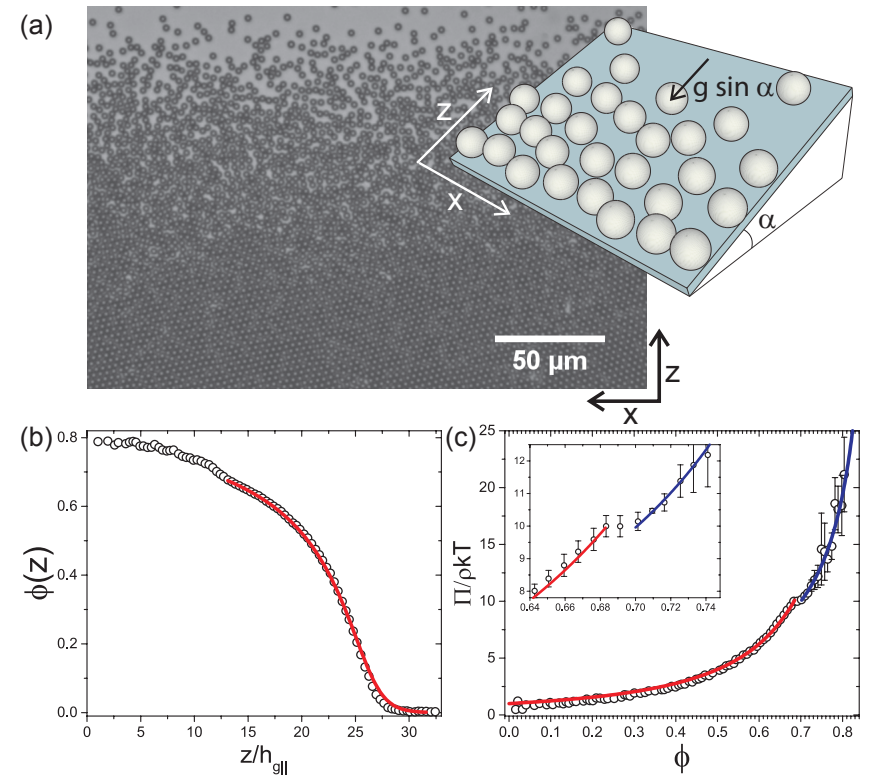

Figure 1. The sedimentation-diffusion equilibrium for $2 \mathrm{D}$ colloidal hard spheres. (a) A typical experimental image of the system in sedimentation-diffusion equilibrium for a tilt angle of $\alpha=0.56^{\circ}$. Inset, a schematic diagram of the experimental geometry showing the effect of tilting the sample by a small angle, $\alpha$, and the resultant in-plane component of gravity. (b) The density profile, $\phi(z)$, as a function of the height, $z$, rescaled by the in-plane gravitational height, $h_{g \|}$. The data shown is averaged over samples at six different values of $\alpha$, with error bars, arising from the standard deviation of this average, smaller than the symbol size. The solid red line indicates the prediction for the density profile in the liquid phase from scaled particle theory [33]. (c) The equation of state $\Pi / \rho k_{\mathrm{B}} T$, averaged over six different values of $\alpha$, with error bars as in (b). The inset shows an expanded view of the behaviour of the equation of state in the region of the discontinuity. The solid red line gives the prediction of scaled particle theory for the range of area fractions characteristic of a liquid, $\Pi / \rho k_{\mathrm{B}} T=1 /(1-\phi)^{2}$. As a guide to the eye, the solid blue line shows a semi-empirical fit to the behaviour at high $\phi$ of $\Pi / \rho k_{\mathrm{B}} T=a /\left(\phi_{c p}-\phi\right)[34]$, where $\phi_{c p}$ is the area fraction at close packing $\left(\phi_{c p} \approx 0.91\right)$.

Supplemental Material [37] for experimental images of the monolayer at all tilt angles). From the tracked particle positions, we determine the density profiles, $\phi(z)$, which are linked to the equation of state as

$$
\frac{\Pi}{\rho k_{\mathrm{B}} T}=\frac{1}{h_{g \|}} \frac{1}{\phi(z)} \int_{z}^{\infty} \phi\left(z^{\prime}\right) \mathrm{d} z^{\prime},
$$

where $\Pi$ is the osmotic pressure, $\rho$ the number density, $k_{\mathrm{B}}$ the Boltzmann constant and $T$ the temperature. As the experimental equation of state in the liquid phase is known to be accurately described by scaled particle theory (SPT) [33, 35], we obtain values of $h_{g \|}$ by calculating the $\phi(z)$-dependent part of Eq. (1) from the experimental density profiles and then comparing this result in the liquid phase with the SPT equation of state. Importantly, this allows the tilt angle, $\alpha$, to be determined directly from the experimental data via $h_{g \|}$, which thus acts as a very sensitive internal calibration mechanism (see Supplemental Material [37]).

The density profiles as a function of the height, $z$, rescaled by $h_{g \|}$, and the full equations of state each fall onto a single curve for systems at different tilt angles and Figs. $1 \mathrm{~b}$ and $\mathrm{c}$ show the density profile and equation of state averaged over the six tilt angles considered. Note that the individual density profiles and equations of state do not vary with tilt angle (see Supplemental Material [37]), which implies that we observe consistent results despite $h_{g \|}$ changing by almost an order of magnitude. Also shown are the SPT expressions for these quantities in the liquid phase, which are in excellent agreement with the experimental data. Strikingly, the equation of state exhibits a discontinuity at $\phi \approx 0.68$, shown more clearly in the inset of Fig. 1c. This is a clear signature of a firstorder transition and the width of the coexistence region can be roughly estimated from the equation of state as $\Delta \phi \approx 0.02$, which is in agreement with the width of the liquid-hexatic coexistence region found in simulations of hard disks [11-14]. The equation of state provides no evidence for another first-order transition at higher area fractions.

Next, to characterise the nature of the different phases, we calculate the height-resolved bond-orientational correlation function in time, $g_{6}(t)$, and the modified Lindemann parameter, $\gamma_{L}(t)$ [20](see Supplemental Material [37]). While $g_{6}(t)$ probes the bond-orientational order, allowing the liquid and hexatic phases to be distinguished, the long-time limit of $\gamma_{L}(t)$ probes the translational order $[20,29]$, which enables the transition between the hexatic and crystal phases to be identified. In Fig. 2a we show the behaviour of $g_{6}(t)$ at a wide range of area fractions, i.e. different heights, for the sample tilted by $\alpha=0.25^{\circ}$. As the area fraction increases, there is a clear change from an exponential decay at low $\phi$, characteristic of a liquid, to $g_{6}(t)$ attaining a constant value at high $\phi$ as expected for a crystal. Crucially, however, at intermediate values of $\phi$, the decay is algebraic, $g_{6}(t) \sim t^{-\eta_{6} / 2}$, characteristic of a hexatic phase. The corresponding measurement of the modified Lindemann parameter for the same sample is shown in Fig. 2b. Upon increasing area fraction, $\gamma_{L}(t)$ exhibits the transient subdiffusive behaviour at intermediate times as expected for a hard disk system upon increasing area fraction [36, 39]. However, there is a systematic change from a linear longtime behaviour at low area fractions, characteristic of the liquid and hexatic phases, to a plateau at high area fractions, characteristic of a crystal.

We further quantify the long-time behaviour of both $g_{6}(t)$ and $\gamma_{L}(t)$ by considering the $\phi$-dependence of the exponents $\eta_{6} / 2$ for $g_{6}(t) \sim t^{-\eta_{6} / 2}$ and $\beta$ for $\gamma_{L}(t) \sim t^{\beta}$. For $g_{6}(t), \eta_{6}$ is predicted to be undefined in the liquid, smaller than $1 / 4$ in the hexatic phase and zero in the 

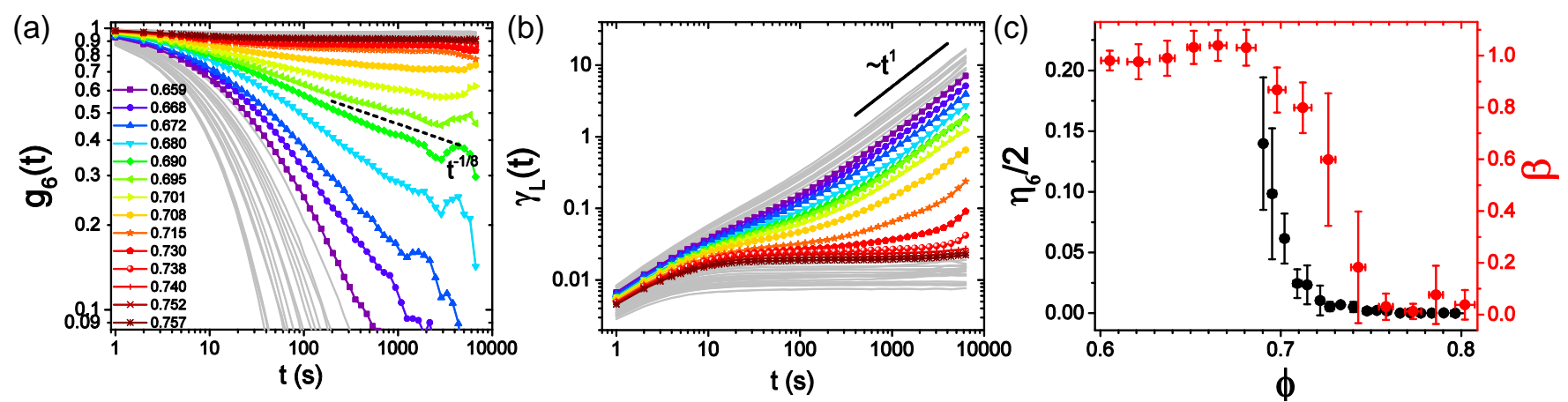

Figure 2. Characterisation of the liquid, hexatic and crystal phases. (a) The height-resolved bond-orientational correlation function in time, $g_{6}(t)$, for the sample at $\alpha=0.25^{\circ}$ for all bins across the whole range of area fractions. Note that a legend is only provided for the data between $0.65<\phi<0.76$. (b) The corresponding height-resolved modified Lindemann parameter, $\gamma_{L}(t)$, for the same sample. The legend in panel (a) also applies to panel (b). (c) The variation in the exponents, $\eta_{6} / 2$, and $\beta$ with $\phi$ in the region of the transitions. Error bars indicate the standard deviation of the average over samples at six different tilt angles.

crystal $[17,22]$. For $\gamma_{L}(t), \beta$ is unity in the liquid and hexatic phases and zero in the crystal [20]. Both sets of exponents, averaged over the six samples at different tilt angles, $\alpha$, are plotted in Fig. 2c for $0.60<\phi<0.80$. Here, $\eta_{6} / 2$ is close to zero at high $\phi$ and increases to a small but finite value at $\phi \approx 0.70$. The exponent $\beta$ is also close to zero at high $\phi$ but increases towards unity at $\phi \approx 0.73$. The fact that the changes in the values of the two exponents with decreasing $\phi$ do not coincide, implies that first the translational order is lost at $\phi \approx 0.73$ and then the bond-orientational order at $\phi \approx 0.70$. Thus, the region between area fractions of approximately 0.70 and 0.73 displays exponents consistent with the presence of a hexatic phase, which corresponds to a width of approximately $2 h_{g \|}$ (see Fig. $1 b$ and Supplemental Material [37]). Crucially, we find that the behaviour of the exponents does not depend on the tilt angle (see Supplemental Material [37]), again confirming that the observed phase behaviour is consistent across all tilt angles. Notably, KTHNY theory predicts that the hexatic phase becomes unstable at $\eta_{6} / 2=0.125[17,20,22,29]$, consistent with our value of $\eta_{6} / 2$ at $\phi \approx 0.70$. Furthermore, if the range of area fractions we attribute to the hexatic phase is compared to the position of the discontinuity in the equation of state, we can infer that the first-order transition is between the liquid and hexatic phases.

The discontinuity in the equation of state suggests a coexistence region of $\Delta \phi \approx 0.02$ for the first-order liquidhexatic transition. Yet we note that the error in directly determining the area fraction from microscopy can be relatively large, with even a small uncertainty in the particle positions or diameter leading to variations in $\phi$ comparable in size to the coexistence region [40]. However, a first-order liquid-hexatic transition is associated with a well-defined interface between the two phases, which will display thermal interface fluctuations. Crucially, these fluctuations are directly related to the width of the coex- istence region $\Delta \phi$ (see Supplemental Material [37]). To localise the interface between the bond-orientationally disordered liquid phase and the bond-orientationally ordered hexatic phase, we compute the hexagonal bondorientational order parameter, $\psi_{6}$, for all particles, and from this the local orientation, $\theta_{i}=\arg \left(\psi_{6, i} / 6\right)$, which varies between $0^{\circ}$ and $60^{\circ}$ due to the hexagonal symmetry (see Supplemental Material [37]). Fig. 3a-d show Voronoi constructions for samples at four different values of $\alpha$, where the Voronoi cells have been coloured according to $\theta_{i}$. These plots clearly show that there is a well defined liquid-hexatic interface, which allows the interface height, $h(x, t)$, to be determined in each frame using tangent hyperbolic fits to $\theta_{i}(z)$ for each bin along the $x$ direction (see Supplemental Material [37]). Note that localising the interface in this manner requires no arbitrary assumptions to be made about the value of $\theta_{i}$ in each phase. The interfaces obtained by this procedure are also shown in Fig. 3a-d. Importantly, the amplitude of the interface fluctuations, as quantified by root mean square width, $\sqrt{\left\langle h^{2}\right\rangle}$, is always less than the width of the hexatic phase $\left(\approx 2 h_{g \|}\right)$ and this difference strongly increases with decreasing tilt angle, as $2 h_{g \|} \sim 1 / \alpha$ and $\sqrt{\left\langle h^{2}\right\rangle} \sim 1 / \alpha^{1 / 4}$ (see Supplemental Material [37]).

The thermal fluctuations of the interface are then analysed by computation of the spatial height-height correlation function, $\left.g_{h}(x)\right)=\left\langle\left[h\left(x_{0}\right)-\bar{h}\left(x_{0}\right)\right]\left[h\left(x_{0}+x\right)-\bar{h}\left(x_{0}+\right.\right.\right.$ $x)]\rangle_{x_{0}, t}$, where $\bar{h}(x)$ is the mean interface height for all frames [41]. From capillary wave theory (see Supplemental Material [37]), this function is predicted to decay as [41]

$$
g_{h}(x)=\frac{k_{B} T}{2 \Gamma} L_{c} \exp \left(-x / L_{c}\right),
$$

where $L_{c}=\sqrt{\Gamma / \Delta \tilde{\rho} g \sin \alpha}$ is the capillary length, $g$ the acceleration due to gravity, $\Delta \tilde{\rho}=\rho_{\text {hex }}-\rho_{\text {liq }}$ the mass density difference between the hexatic and liquid phases 

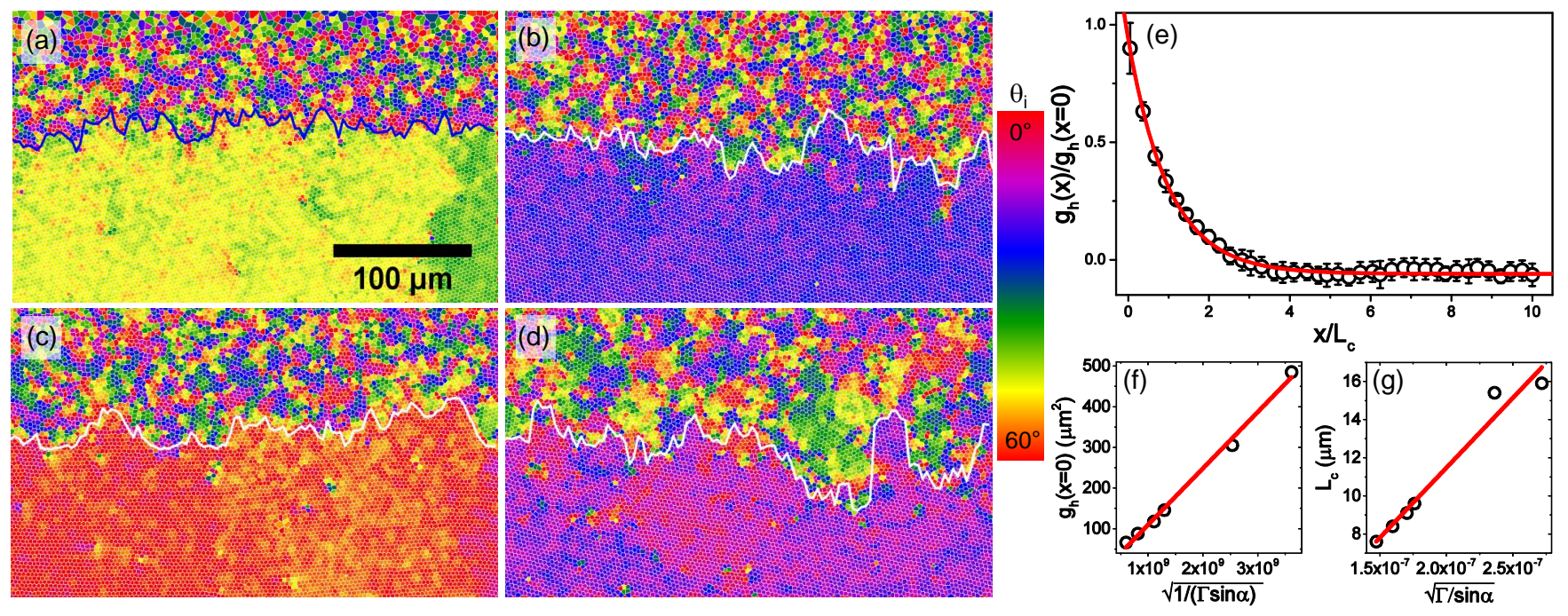

Figure 3. Analysis of the liquid-hexatic interface and coexistence region. (a)-(d) Typical Voronoi plots with cells coloured by $\theta_{i}$ for systems at: (a) $\alpha=0.56^{\circ}$, (b) $\alpha=0.35^{\circ}$, (c) $\alpha=0.25^{\circ}$ and (d) $\alpha=0.067^{\circ}$. (e) The mean height-height correlation function, $g_{h}(x)$, rescaled by $g_{h}(x=0)$, for the six samples considered. Note that the $x$-axis is rescaled by the capillary length, $L_{c}$. The solid red line shows an exponential decay, according to Eq. (2) and error bars indicate the standard deviation of the average over samples at six different tilt angles. (f) and (g): Plots used to determine the coexistence gap $\Delta \phi$ exhibiting the linear dependence of (f) $g_{h}(x=0)$ with $\sqrt{1 / \Gamma \sin \alpha}$ and (g) $L_{c}$ with $\sqrt{\Gamma / \sin \alpha}$.

and $\Gamma$ the anisotropic interfacial stiffness. In Fig. 3e we show $g_{h}(x) / g_{h}(x=0)$ as a function of $x / L_{c}$, averaged over the six values of $\alpha$, and find excellent agreement with an exponential decay as predicted by Eq. (2). The two independent parameters, $g_{h}(x=0)=k_{B} T L_{c} /(2 \Gamma)$ and $L_{c}$, extracted from fitting Eq. (2) to the data for each tilt angle $\alpha$, both depend upon $\Gamma$ and $\Delta \tilde{\rho}$. By first resolving the value of $\Gamma$ for each sample (see Supplemental Material [37]), we are then able to plot the variation of $g_{h}(x=0)$ and $L_{c}$ as functions of $\sqrt{1 / \Gamma \sin \alpha}$ and $\sqrt{\Gamma / \sin \alpha}$, respectively, as shown in Figs. 3f and g. Importantly, the gradients of these linear plots are directly related to the mass density difference between the liquid and hexatic phases, $\Delta \tilde{\rho}$, and as such give a quantitative measure of the width of the coexistence gap, $\Delta \phi$. From this analysis we find values of $\Delta \phi$ to be 0.022 and 0.019 from Figs. 3f and $g$, which is in excellent agreement with both the apparent coexistence gap in our equation of state and results from simulations [11-14].

From our experimental results the following picture of the phase behaviour of hard disks emerges. The exponents associated with time-correlation functions clearly indicate the presence of three phases, liquid, hexatic and crystal. The liquid-hexatic transition is found to be first order, with a coexistence region of $\phi \approx 0.68-0.70$. A quantitative measure of the size of this coexistence gap is obtained by analysing the fluctuations of the interface between the liquid and hexatic phases and independently found to be $\Delta \phi \approx 0.02$. The hexatic phase is observed for $0.70<\phi<0.73$, with the hexatic-crystal transition at $\phi \approx 0.73$. The absence of a second discontinuity in the equation of state at this area fraction indicates that this transition is continuous. This experimentally established phase behaviour is in excellent agreement with that observed in simulations [11], especially if it is noted that very small out-of-plane fluctuations shift the phase transitions to lower $\phi[13]$. We also note that our results differ from those obtained for soft dipolar colloidal systems [19-23], consistent with the dependence of the melting scenario on the details of the particle interactions [14]. With this study we therefore provide a definitive experimental elucidation of the full phase behaviour of hard disks and thereby the fundamental cornerstone for the further understanding and development of 2D materials.

Acknowledgements. We thank Michael Juniper, Francois Lavergne, Daan Frenkel and Jürgen Horbach for useful discussions. The EPSRC and ERC (ERC Starting Grant 279541-IMCOLMAT) are acknowledged for financial support.

[1] B. J. Alder and T. E. Wainwright, J. Chem. Phys. 27, 1208 (1957).

[2] W. G. Hoover and F. H. Ree, J. Chem. Phys. 49, 3609 (1968).

[3] J. K. Percus, ed., The Many-Body Problem (Wiley, 1963).

[4] P. N. Pusey and W. V. Megen, Nature 320, 340 (1986).

[5] B. J. Alder and T. E. Wainwright, Phys. Rev. 127, 359 (1962).

[6] J. Lee and K. J. Strandburg, Phys. Rev. B 46, 11190 (1992). 
[7] J. A. Zollweg and G. V. Chester, Phys. Rev. B 46, 11186 (1992).

[8] J. F. Fernández, J. J. Alonso, and J. Stankiewicz, Phys. Rev. Lett. 75, 3477 (1995).

[9] A. Jaster, Phys. Rev. E. 59, 2594 (1999).

[10] K. Binder, S. Sengupta, and P. Nielaba, J. Phys.: Condens. Matter 14, 2323 (2002).

[11] E. P. Bernard and W. Krauth, Phys. Rev. Lett. 107, 155704 (2011).

[12] M. Engel, J. A. Anderson, S. C. Glotzer, M. Isobe, E. P. Bernard, and W. Krauth, Phys. Rev. E 87, 042134 (2013).

[13] W. Qi, A. P. Gantapara, and M. Dijkstra, Soft Matter 10, 5449 (2014).

[14] S. C. Kapfer and W. Krauth, Phys. Rev. Lett. 114, $035702(2015)$.

[15] J. M. Kosterlitz and D. J. Thouless, J. Phys. C: Solid State Phys. 6, 1181 (1973).

[16] B. I. Halperin and D. R. Nelson, Phys. Rev. Lett. 41, 121 (1978).

[17] D. R. Nelson and B. I. Halperin, Phys. Rev. B 19, 2457 (1979).

[18] A. P. Young, Phys. Rev. B 19, 1855 (1979).

[19] K. Zahn, R. Lenke, and G. Maret, Phys. Rev. Lett. 82, 2721 (1999).

[20] K. Zahn and G. Maret, Phys. Rev. Lett. 85, 3656 (2000).

[21] H. H. Von Grünberg, P. Keim, and G. Maret, in Soft Matter (Wiley, 2007), vol. 3, pp. 40-83.

[22] P. Keim, G. Maret, and H. H. von Grünberg, Phys. Rev. E 75, 031402 (2007).

[23] U. Gasser, C. Eisenmann, G. Maret, and P. Keim, ChemPhysChem 11, 963 (2010).

[24] P. Bladon and D. Frenkel, Phys. Rev. Lett. 74, 2519 (1995).

[25] J. A. Anderson, J. Antonaglia, J. A. Millan, M. Engel, and S. C. Glotzer, arXiv:1606.00687 (2016).

[26] S. Deutschländer, T. Horn, H. Löwen, G. Maret, and P. Keim, Phys. Rev. Lett. 111, 098301 (2013).
[27] W. Qi and M. Dijkstra, Soft Matter 11, 2852 (2015).

[28] M. A. Bates and D. Frenkel, Phys. Rev. E 61, 5223 (2000).

[29] Y. Han, N. Y. Ha, A. M. Alsayed, and A. G. Yodh, Phys. Rev. E 77, 041406 (2008).

[30] C. A. Murray and D. H. Van Winkle, Phys. Rev. Lett. 58, 1200 (1987).

[31] P. Karnchanaphanurach, B. Lin, and S. A. Rice, Phys. Rev. E. 61, 4036 (2000).

[32] A. H. Marcus and S. A. Rice, Phys. Rev. Lett. 77, 2577 (1996).

[33] E. Helfand, H. L. Frisch, and J. L. Lebowitz, J. Chem. Phys. 34, 1037 (1961).

[34] Z. W. Salsburg and W. W. Wood, J. Chem. Phys. 37, 798 (1962).

[35] A. L. Thorneywork, R. Roth, D. G. A. L. Aarts, and R. P. A. Dullens, J. Chem. Phys. 140, 161106 (2014).

[36] A. L. Thorneywork, R. E. Rozas, R. P. A. Dullens, and J. Horbach, Phys. Rev. Lett. 115, 268301 (2015).

[37] See Supplemental Material at http://link.aps.org/ supplemental/xx.xxxx/PhysRevLett.xxx.xxxxxx, which includes Refs. [42-45], for a description of the colloidal model system, the data analysis and the data for the system at all tilt angles.

[38] J. C. Crocker and D. G. Grier, J. Colloid Interface Sci. 179, 298 (1996).

[39] A. L. Thorneywork, D. G. A. L. Aarts, J. Horbach, and R. P. A. Dullens, Phys. Rev. E 95, 012614 (2017).

[40] W. C. K. Poon, E. R. Weeks, and C. P. Royall, Soft Matter 8, 21 (2012).

[41] T. O. E. Skinner, D. G. A. L. Aarts, and R. P. A. Dullens, Phys. Rev. Lett. 105, 168301 (2010).

[42] S. Pronk and D. Frenkel, Phys. Rev. E 69, 066123 (2004).

[43] D. G. A. L. Aarts, M. Schmidt, and H. N. W. Lekkerkerker, Science 304, 847 (2004).

[44] J. Hoyt, Z. Trautt, and M. Upmanyu, Math. Comput. Simul. 80, 1382 (2010).

[45] V. D. Nguyen, Z. Hu, and P. Schall, Phys. Rev. E 84, 011607 (2011). 